\title{
Ueber Normirung der linearen Differentialgleichungen zweiter Ordnung.
}

Von

FeLIx KLenv in Göttingen.

Anschliessend an die vorangehende Mittheilung des Hrn. Pick möchte ich hier verwandte Betrachtungen zum Abdruck bringen, welche ich in den Göttinger Nachrichten vom März dieses Jahres in einer der Theorie der La mé'schen Functionen gewidmeten Arbeit publicirte.*) Es heisst dort u. a.:

„Wir fragen zunächst nach der zweckmässigsten Definition der zu einem $n$-fach ausgedehnten Raume $\left(R_{n}\right)$ gehörigen Laméschen Differentialgleichung. In dieser Hinsicht beginnt man herkömmlicher Weise mit dem System der confocalen Flächen zweiten Grades

$$
\frac{x_{1}^{2}}{\lambda-e_{1}}+\cdots+\frac{x_{n}^{2}}{\lambda-e_{n}}=1
$$

und findet durch bekannte Umformungen der Potentialgleichung die Lamé'sche Gleichung in der Gestalt:

wo

$$
\frac{d^{2} E}{d t^{2}}=\left(A \lambda^{n-2}+B \lambda^{n-3}+\cdots+N\right) E,
$$

$$
t=\int \frac{d \lambda}{\sqrt{f(\lambda)}}, \quad f(\lambda)=\left(\lambda-e_{1}\right) \cdots\left(\lambda-e_{n}\right)
$$

ich habe schon bei früherer Gelegenheit hervorgehoben $* *$, dass es zweckmässig ist, die hier auftretenden Constanten $A, B, \ldots, N$ zunächst als unbeschränkt veränderlich zu betrachten und dadurch der gewöhnlichen

*) Diese Arbeit reproducirt verschiedene Resultate, welche ich während des Wintersemesters 1889-1890 in einer Vorlesung über Lamé'sche Functionen abgeleitet habe. Es handelt sich dabei insbesondere auch um die Oscillationen, welche die Lösungen der Lamé'schen Differentialgleichung in gegebenen Intervallen ausführen; ich hoffe hierauf in diesen Annalen bei anderer Gelegenheit ausführlich eingehen zu können.

**) Math. Ann. Bd. 18 (1881): Ueber Körper, welche von confocalen Flächen zweiten Grades begränzt sind. 
Begriffsbestimmung der Lamé'schen Functionen gegenüber eine Erweiterung eintreten zu lassen*). Nun kann man aber den durch (1) gegebenen Ausgangspunkt beanstanden. In der Potentialtheorie, wo fortgesetzt Transformationen durch reciproke Radien in Betracht zu ziehen sind, ist das System der confocalen Flächen zweiten Grades kein wirklich allgemeines Orthogonalsystem; als solches erscheint vielmehr erst das von Darboux und Moutard im Jahre 1864 aufgestellte System der confocalen Cycliden, ein System von Flächen vierter Ordnung, das sich bei Verwendung überzähliger, homogener Coordinaten $\left(x_{1} \ldots x_{n+2}\right)$ [sogenannter polysphärischer Coordinaten] durch die zwei Gleichungen darstellen lässt:

$$
\sum_{1}^{n+2} x_{x}^{2}=0, \quad \sum_{x}^{n+2} \frac{x_{x}^{2}}{\lambda-e_{x}}=0 .
$$

Herr Wangerin ist der Erste gewesen, der nachwies, dass man unter Zugrundelegung eines solchen Cyclidensystems in der That eine Theorie ganz ähnlich der Lamé'schen aufbauen kann**). Seine Rechnungen beziehen sich allerdings nur auf $n=3$; es ist aber nicht schwer, sein Resultat auf beliebiges $n$ zu übertragen; es tritt dann an Stelle von (2) die folgende Differentialgleichung:

(5) $\frac{d^{2} E}{d t^{2}}=\left(\frac{4-n^{2}}{16} \cdot \lambda^{n}+\frac{n^{2}-2 n}{16} \cdot \sum_{1}^{n+2} e_{\alpha} \cdot \lambda^{n-1}+A \lambda^{n-2}+\cdots+N\right) E$,

wo $t$ wiederum das Integral bezeichnet:

$$
t=\int \frac{d \lambda}{\sqrt{f(\lambda)}}
$$

$f(\lambda)$ aber die Function $(n+2)^{\text {ten }}$ Grades bedeutet:

$$
f(\lambda)=\left(\lambda-e_{1}\right) \ldots\left(\lambda-e_{n+2}\right) .
$$

Offenbar kann man statt (5) auch schreiben:

$$
\frac{d^{2} E}{d t^{2}}=\left(\frac{2-n}{16(n+1)} f^{\prime \prime}(\lambda)+a \lambda^{n-2}+\cdots+n\right) E,
$$

wo nun die $a, \ldots, n$ beliebig. Es scheint fast, als habe man diesen Gleichungen (5), (8) bisher nur wenig Bedeutung beigelegt. Sicher kann man die Gleichung (2) als Specialfall derselben auffassen (der

*) Lamé und Heine bestimmen $A, B, \ldots$ bekanntlich so, dass eine Particularlösung von (2) algebraisch wirả; Hermite führt bei seinen allgemeineren, auf $n=3$ bezüglichen Untersuchungen für $A$ immer noch den besonderen im algebraischen Falle eintretenden Werth $\frac{n(n+1)}{4}$ ein (wo $n$ eine positive ganze Zahl) und lässt dann freilich $B$ beliebig.

**) Journal für Mathematik, Bd, 82, 1876. Vergl. auch Darboux in den Comptes Rendus der Pariser Akademie, 1876, II. 
entsteht, wenn man $e_{n+1}^{j}$ und $e_{n+2}$ unendlich werden lässt), aber es lag näher, (5) oder (8) als besonderen Fall derjenigen Gleichungen (2) zu deuten, die dem Raume von $(n+2)$ Dimensionen entsprechen. Und dieser Fall schien kein besonderes Interesse darzubieten, weil man die $(n-1)$ bei ihm noch zur Verfügung stehenden Constanten keineswegs so bestimmen kann, dass eins der zugehörigen $E$ algebraisch wird. Auch hat die Form der neuen Differentialgleichung zunächst wenig Ansprechendes. Singuläre Punkte hat dieselbe, wie dies natürlich scheint, bei $\lambda=e_{1}, \ldots, e_{n+2}$; aber auch $\lambda=\infty$, d. h. ein Werth, der für das Cyclidensystem (4) vom geometrischen Standpunkte aus ohne jede specifische Bedeutung ist, erscheint als singulärer Punkt. Die zu $e_{1} \ldots e_{n+2}$ gehörigen Exponenten berechnen sich dabei als $\frac{1}{2}$ und 0 , die zu $\infty$ gehörigen als

$$
\frac{n-2}{4}+1 \text { und } \frac{n-2}{4} \text {. }
$$

Inzwischen gelingt es durch eine unbedeutende formale Abänderung unsere Gleichung in ganz anderem Lichte erscheinen zu lassen. Die bei $\lambda=\infty$ auftretenden Exponenten leiten auf den richtigen Weg. Man setze nämlich, homogen machend,

und schreibe

$$
\lambda=\lambda_{1}: \lambda_{2}
$$

$$
E(\lambda)=\frac{n-2}{\lambda_{2}^{4}} \cdot F\left(\lambda_{1}, \lambda_{2}\right),
$$

wo $F$ jetzt eine homogene Function (eine Form) von $\lambda_{1}, \lambda_{2}$ vom Grade

$$
\frac{2-n}{4}
$$

sein wird, die ich gleich als Lamés sche Form bezeichnen will. Sei ferner jetzt unter $f$ die Form $(n+2)^{\text {ten }}$ Grades verstanden:

$$
f=\left(\lambda_{1}-e_{1} \lambda_{2}\right) \ldots\left(\lambda_{1}-e_{n+2} \lambda_{2}\right) \text {. }
$$

Man erhält dann (nach kurzer Umrechnung - mittelst des Euler'schen Theorems) ein Resultat, welches nur noch von der Form $f$ als solcher abhängig ist; man findet nämlich:

$$
(f, F)_{2}=\varphi \cdot F
$$

wo $(f, F)_{2}$ die zweite Ueberschiebung der Formen $f$ und $F$ vorstellt, $\varphi$ aber eine beliebige rationale ganze Frorm $(n-2)^{\text {ten }}$ Grades ist. Dieses Resultat erscheint so einfach, dass man nicht umhin kann, dasselbe überhaupt an die Spitze der Theorie der Lamé'schen Functionen zu stellen und dementsprechend Lamé sche Functionen, oder vielmehr Lamé'sche Formen, des $R_{n}$ geradezu als solche Formen $\left(\frac{2-n}{4}\right)^{\text {ten }}$ Gradtes von $\lambda_{1}, \lambda_{2}$ zu definiren, welche zweimal über eine gegebene $f_{n+2}$ 
geschoben, sich selbst bis auf einen Factor $\varphi_{n-2}$ reproduciren*). Die einzigen singulären Stellen der so definirten $F$ sind, wie bereits angedeutet, die Wurzeln von $f=0$. Sind diese Wurzeln alle getrennt (wie wir bisher stillschweigend voraussetzten), so gehören $\mathrm{zu}$ jeder einzelnen derselben die soeben genannten Exponenten $\frac{1}{2}$ und 0 , rücken aber irgendwo zwei oder mehrere derselben zusammen, so erhält man höhere Exponenten, bez. irreguläres Verhalten. Der gewöhnliche Fall der durch (2) definirten Functionen entsteht, wenn $f$ eine Doppelwurzel erhält (die man dann nach $\lambda=\infty$ wirft). Uebrigens kann man, wenn man $f$ mit beliebig vielfachen Wurzeln ausstatten will und sich vorhält, von der Form $F$ der homogenen Variabelen $\lambda_{1}, \lambda_{2}$ durch Zufügung irgend welcher Factoren zu Functionen von $\lambda$ zurückzugehen, sämmtliche lineare Differentialgleichungen zweiter Ordnung mit rationalen Coefficienten unter (11) rubriciren. Die Lamé'sche Differentialgleichung hat also in der That eine wesentlich allgemeine Bedeutung. Die Differentialgleichung der hypergeometrischen Reihe z. B. entsteht aus (11), wenn man $f$ als eine Form sechsten Grades einführt, die ein volles Quadrat ist. Noch ein weiterer Gesichtspunkt spielt hier herein. Wählt man $f$ wieder als Form sechsten Grades, aber nun mit getrennten Wurzeln, so definirt (11) solche Formen $F$ vom Grade $-\frac{1}{2}$, welche auf dem hyperelliptischen Gebilde $\sqrt{\hat{f}}$ durchaus unverzweigt sind. Man überzeugt sich leicht, dass diese $F$ unter allen Formen, die einer linearen Differentialgleichung zweiter Ordnung mit rationalen Coefficienten genügen, die einzigen sind, welche die genannte Eigenschaft besitzen. Nicht so bei höherem Grade von $f$. Sei $n=2 p$ gesetzt, $p$ aber als $>2$ genommen, so werden die allgemeinsten zum hyperelliptischen Gebilde $\sqrt{f_{2 p+2}}$ gehörigen unverzweigten $F^{\prime}$ durch die Gleichung geliefert:

$$
(f, F)_{2}=\left(\varphi_{2 p-2}+\psi_{p-3} \sqrt{f}\right) \cdot F,
$$

die sich von (11) durch das Glied mit $\sqrt{f}$ unterscheidet. Die Anzahl der hier in $\varphi$ und $\psi$ zusammen enthaltenen willkürlichen Constanten ist $3 p-3$, der Grad von $F$ gleich $-\frac{p-1}{2}$. Die Theorie von (11)

*) Das gleiche Mittel der Einführung homogener Variabler gebranchen bereits in demselben Sinne Pick und Halphen (Berichte der Wiener Akademie von 1887; Traité des fonctions elliptiques, Bd. II, 1888); nur handelt es sich bei ihnen um eine ganz specielle Untersuchang, nämlich um eine geschickte (nur in diesem Falle mögliche) Transformation höheren Grades der für $n=3$ geltendén Differentialgleichang (2). Es ist wohl kein Zweifel, dass die geeignete Verwendung homogener Variabler in der Theorie der linearen Differentialgleichangen noch vielfache Vereinfachungen nach sich ziehen wird. 
wird als Vorbereitung der allgemeinen Theorie der Gleichungen (12) erachtet werden können*)."

Ich habe diesen Erläuterungen, die ich, ebenso wie die beigesetzten Bemerkungen, ungeändert dem genannten Aufsatze entnahm, hier nur wenige Worte hinzuzufügen, die sich auf bestimmte Integrale beziehen. Bekanntlich hat die Theorie der bestimmten Integrale in den letzten Jahren dadurch einen bemerkenswerthen neuen Aufschwung genommen, dass man sich entschloss, die Integrale ganz allgemein durch geschlossene Wege im complexen Gebiete (auf denen die zu integrirende Function ihren Anfangswerth wieder annimmt) zu definiren, - wobei denn alle die Ausnahmen, welche die ältere Theorie wegen des Unendlichwerdens der zu integrirenden Function statuiren musste, oder auch die Kunstgriffe, zu denen sie in solchen Fällen ihre Zuflucht nahm, von selbst in Wegfall kommen**). Ich möchte nun darauf hinweisen, dass die Darstellung dieser Verhältnisse noch um Vieles eleganter wird, wenn

*) Sind $F_{1}, F_{2}$ irgend zwei Particularlösungen von (12), so ist der Quotient $\eta=F_{1}: F_{2}$ eine auf dem hyperelliptischen Gebilde unverzweigte ,Function“, welche bei jedem geschlossenen Umlaufe über die zu $\sqrt{f}$ gehörige Riemann'sche Fläche hin sich in der Gestalt $\frac{\alpha \eta+\beta}{\gamma \eta+\delta}$ reproducirt. Dass es auf jedem algebraischen Gebilde, dessen $p>1, \infty^{3} p-3$ wesentlich verschiedene (durch ihre Differentialgleichnng unterschiedene) derartige $\eta$-Functionen giebt, ist bekannt (vergl. z. B. meine "Neuen Beiträge zur Riemann'schen Functionentheorie " im 21 ten Bande der Math. Annalen, 1882); man hatte aber bisher, so viel ich weiss, diese $\eta$ noch nicht in zwei Formen $\boldsymbol{F}_{1}, \boldsymbol{F}_{2}$ als Zähler und Nenner derart gespalten, dass $\boldsymbol{F}_{1}$ und $F_{2}$ für sich genommen auf dem algebraischen Gebilde gleichfalls unverzweigt sind. Dies gelingt aber sofort allgemein, wenn man diejenigen Erläuterungen heranzieht, die ich über die anf beliebigen algebraischen Gebilden existirenden Formen nenerdings gegeben habe (Zur Theorie der Abel'schen Functionen, Math. Annalen Bd. 36). Der Grad der betr. $F_{1}, F_{2}$ in den zum Gebilde gehörigen Formen $\varphi$ ist allemal gleich $-\frac{1}{2},-$ in Uebereinstimmung mit dem, was im Texte speciell für hyperelliptische Gebilde bemerkt ist.

**) Vergl. Camille Jordan in Bd. III seines Cours d'analyse, p. $241 \mathrm{ff}$. (1887), Nekrass off in der "Mathematischen Sammlung" der Moskauer mathematischen Gesellschaft von 1887 an [die genaneren Citate vergl. bei Pochhammer in Bd. 37 dieser Annalen, p. 543], Pochhammer in den Bänden 35, 36, 37 dieser Annalen. - Die Idee selbst geht wohl unzweifelhaft auf Riemann zurück, der ja die Perioden der Abel'schen Integrale in entsprechender Weise definirte (durch geschlossene Wege auf den zugehörigen Riemann'schen Flächen). Inzwischen finden sich, was den allgemeinen Ansatz und seine Bedentung für die Theorie der gewöhnlich betrachteten bestimmten Integrale angeht; in Riemanns publicirten Arbeiten nur Andeutangen (vergl. Werke, pag. 77, 137, 404), an welche dann zunächst Hankel (Schlömilch's Zeitschrift Bd. 9, 1864) und Thomae (ebenda, Bd. 14, 1869) anknüpften, die aber immer noch an schleifenförmigen Integrationswegen festhielten, $d . h$, an Integrationswegen, die, von einem bestimmten Punkte auslaufend zu eben diesem bestimmten Pankte zurückführen, womit die ganze Allgemeinheit, die hier anzustreben ist, sozusagen erst zur Hälfte erreicht ist. 
man sich entschliesst, auch hier homogene Veränderliche anzuwenden. Unter $(z a) \ldots$ die Determinanten $\left(z_{1} a_{2}-z_{2} a_{1}\right)$ etc. verstanden, wird man zunächst Integrale der folgenden Art betrachten:

$$
\int(z a)^{\alpha}(z b)^{\beta} \cdots(z x)^{\nu}(z d z),
$$

wo $\alpha+\beta+\cdots+\nu$ natürlich gleich $(-2)$ zu nehmen ist und die Integration über solche geschlossene Curven der $z$-Ebene zu nehmen ist, wie sie Herr Pochhammer als Doppelumläufe bezeichnet. Ist die Zahl $n$ der hier auftretenden singulären Punkte gleich 2, so verschwindet das Integral identisch; ist sie gleich 3 , so hat man im wesentlichen ein Euler'sches Integral erster Gattung (bei welchem dann die Gleichberechtigung der drei Exponenten $\alpha ; \beta, \gamma$, die sonst auf indirectem Wege erschlossen wird, unmittelbar hervortritt). Für $n=4$ kommen diejenigen Integrale, durch welche man die Differentialgleichung der hypergeometrischen Reihen integrirt, beziehungsweise die Riemann'sche $P$-Function darstellen kann. Auch bei letzterer ist selbstverständlich der Gebrauch homogener Variabler indicirt. Riemann unterwirft die Exponenten seiner Function

$$
P\left|\begin{array}{llll}
\alpha & b & c & \\
\lambda^{\prime} & \mu^{\prime} & \nu^{\prime} & x \\
\lambda^{\prime \prime} & \mu^{\prime \prime} & \nu^{\prime \prime} &
\end{array}\right|
$$

bekanntlich der Bedingung, als Summe die Eins zu geben:

$$
\lambda^{\prime}+\lambda^{\prime \prime}+\mu^{\prime}+\mu^{\prime \prime}+\nu^{\prime}+\nu^{\prime \prime}=1,
$$

und an dieser Bedingung ändert sich nichts, wenn wir $P$ mit einem geeigneten Factor multipliciren, der nur bei $x=a, b, c$ unendlich wird, beziehungsweise verschwindet:

$$
\begin{aligned}
& (x-a)^{l} \cdot(x-b)^{m} \cdot(x-c)^{n} \cdot P\left|\begin{array}{llll}
a & b & c & \\
\lambda^{\prime} & \mu^{\prime} & \nu^{\prime} & x \\
\lambda^{\prime \prime} & \mu^{\prime \prime} & \bar{\nu}^{\prime \prime} &
\end{array}\right| \\
& =P\left|\begin{array}{ccc}
a & b & c \\
\lambda^{\prime}+l & \mu^{\prime}+m & \nu^{\prime}+n \\
\lambda^{\prime \prime}+l & \mu^{\prime \prime}+m & \nu^{\prime \prime}+n
\end{array}\right|
\end{aligned}
$$

In der That muss ja hier $l+m+n=0$ genommen werden, weil anderenfalls der Punkt $x=\infty$ singulär werden würde. Indem wir homogene Variable einführen, können wir die Bedingung (15) bei Seite schieben. Schreiben wir nämlich statt der in (16) auftretenden Differenzen $(x-a)$ etc. entsprechende Determinanten $\left(x_{1} a_{2}-x_{2} a_{1}\right), \ldots$, so hindert Nichts, eine Function $\Pi$ der homogenen Veränderlichen $x_{1}, x_{2}$ (eine Form der $x_{1}, x_{2}$ ) durch die Gleichung zu definiren: 


$$
\Pi=(x a)^{l} \cdot(x b)^{m} \cdot(x c)^{n} \cdot P\left|\begin{array}{llll}
a & b & c & \\
\lambda^{\prime} & \mu^{\prime} & \nu^{\prime} & x \\
\lambda^{\prime \prime} & \mu^{\prime \prime} & \nu^{\prime \prime} &
\end{array}\right|
$$

wo nun die $l, m, n$ beliebig anzunehmen sind. Wir bezeichnen dieses $\Pi$, indem wir für $\lambda^{\prime}+l, \ldots$ wieder $\operatorname{kurz} \lambda^{\prime}, \ldots$ schreiben, mit

$$
\Pi\left|\begin{array}{llll}
a & b & c & \\
\lambda^{\prime} & \mu^{\prime} & \nu^{\prime} & x_{1}, x_{2} \\
\lambda^{\prime \prime} & \mu^{\prime \prime} & \nu^{\prime \prime} &
\end{array}\right|
$$

die Summe $\frac{\lambda^{\prime}+\lambda^{\prime \prime}+\mu^{\prime}+\mu^{\prime \prime}+\nu^{\prime}+\nu^{\prime \prime}-1}{2}$ stellt dann den Grad dar, welchen das $\Pi$ in $x_{1}, x_{2}$ besitzt. Indem wir die $l, m, n$ in (17) zweckmässig wählen, können wir dieses $\Pi$ natürlich in verschiedener Weise normiren. Sei der Kürze halber:

$$
\lambda^{\prime}-\lambda^{\prime \prime}=\lambda, \quad \mu^{\prime}-\mu^{\prime \prime}=\mu, \quad \nu^{\prime}-\nu^{\prime \prime}=\nu .
$$

Dann können wir als Normal- $\Pi$ beispielsweise das folgende betrachten:

$$
\Pi\left|\begin{array}{rrrr}
a & b & c \\
+\frac{\lambda}{2} & +\frac{\mu}{2} & +\frac{\nu}{2} & x_{1}, x_{2} \\
-\frac{\lambda}{2} & -\frac{\mu}{2} & -\frac{\nu}{2} &
\end{array}\right|
$$

oder auch das folgende:

$$
\Pi\left|\begin{array}{llll}
a & b & c & \\
\lambda & \mu & \nu & x_{1}, x_{2} \\
0 & 0 & 0 &
\end{array}\right|
$$

(welches auf 8 Weisen herzustellen ist, weil die $\lambda, \mu, \nu$ in (19), indem man die $\lambda^{\prime}, \lambda^{\prime \prime}$ etc. in ihrer Reihenfolge vertauscht, beliebig im Vorzeichen geändert werden können). Hier ist das durch Formel (20) gegebene $\Pi$, vom Grade $-\frac{1}{2}$ in den $x_{1}, x_{2}$, dasjenige, welches durch die normirte Differentialgleichung bestimmt wird, von der oben die Rede war:

$$
(\Pi, f)_{2}=\varphi \cdot \Pi
$$

wo aun $f=(x a)^{2}(x b)^{2}(x c)^{2}$ zu-nehmen ist und die quadratische Form $\varphi$ in einfacher Weise von den $\lambda, \mu, \nu$ abhängt*). Andererseits ist das durch (21) gegebene $\Pi$ gerade dasjenige, welches unmittelbar durch ein bestimmtes Integral von der Form (13) gegeben wird:

*) Man findet des Näheren:

$$
\begin{aligned}
\varphi=\frac{5}{8}\left\{\left(8 \lambda^{2}+1\right)(a b)(a c)(x b)(x c)\right. & +\left(8 \mu^{2}+1\right)(b c)(b a)(x c)(x a) \\
& \left.+\left(8 v^{2}+1\right)(c a)(c b)(x a)(x b)\right\}
\end{aligned}
$$




$$
\begin{aligned}
& \Pi\left|\begin{array}{cccc}
a & b & c & \\
\lambda & \mu & \nu & x_{1}, x_{2} \\
0 & 0 & 0 &
\end{array}\right| \\
& =\int(z a)^{\frac{-1+\lambda-\mu-\nu}{2}} \cdot(z b)^{\frac{-1+\mu-\nu-\lambda}{2}} \cdot(z c)^{\frac{-1+\nu-\lambda-\mu}{2}} \cdot(z x)^{\frac{-1+\lambda+\mu+\nu}{2}} \cdot(z d z),
\end{aligned}
$$

(das Integral wieder über Doppelumläufe des $z$ erstreckt). Es ist nicht unwichtig, diese Formel so aufzufassen, dass man das Integral geradezu als Definition des linker Hand stehenden $\Pi$ gelten lässt. Man erreicht dann, dass $\Pi$ nicht nur von $x_{1}, x_{2}$, sondern auch von $a_{1}, a_{2}$; $b_{1}, b_{2} ; c_{1}, c_{2}$ wie von den Exponenten $\lambda, \mu, \nu$ in ganz bestimmter Weise abhängt $\left.{ }^{*}\right): \Pi$ erscheint als eine Covariante der 4 Reihen cogredienter binärer Veränderlicher $a_{1}, a_{2} ; b_{1}, b_{2} ; c_{1}, c_{2} ; x_{1}, x_{2}$, es erscheint überdies als ganze Function der Exponenten $\lambda, \mu, \nu$. In letzterem Umstande liegt es begründet, dass man nun hier keinerlei, z. B. ganzzahlige Werthsysteme der $\lambda, \mu, \nu$ bei der Definition auszuschliessen hat; vielmehr sind alle besonderen Verhältnisse, die sich bei einzelnen Werthsystemen $\operatorname{der} \lambda, \mu, \nu$ einstellen mögen, aus der allgemeinen Theorie durch Grenzübergang abzuleiten. - Ich hoffe sehr, dass im Sinne dieser Andeutungen eine zusammenhängende Darstellung sämmtlicher Eigenschaften der hypergeometrischen Function von anderer Seite in nicht zu ferner Zeit veröffentlicht wird; ich selbst habe die Grundzüge einer solchen Ableitung im Sommersemester 1890 vorgetragen.

Schliessen wir aus, dass die $\lambda, \mu, \nu$ rein imaginär sind, so giebt es unter den acht jedesmal zusammengehörigen Fällen (21) einen, dessen $\lambda, \mu, \nu$ in ihrem reellen Theile positiv sind. Die Zweige $\Pi_{1}, \Pi_{2}$ des betreffenden $\Pi$ sind als Formen von $x_{1}, x_{2}$ überall endlich, ohne irgendwo eine gemeinsame Nullstelle zu besitzen. Man wird daher die so particularisirten $\Pi_{1}, \Pi_{2}$ zu Grunde legen, wenn es sich darum handelt, $x_{1}, x_{2}$ rückwärts als Formen der $\Pi_{1}, \Pi_{2}$ darzustellen. Die ist bekanntlich insbesondere dann anzustreben, wenn die $\lambda, \mu, \nu$ den reciproken Werthen dreier reeller ganzer Zahlen $l, m, n$ gleich sind. Indem wir $l, m, n$ gleich als positiv voraussetzen, hat das in Rede stehende $\Pi$ in den $x_{1}, x_{2}$ dann den Grad:

$$
\left(\frac{1}{l}+\frac{1}{m}+\frac{1}{n}-1\right): 2
$$

*) Hiermit dürfte geleistet sein, was Riemann beabsichtigte, als er sich (Werke, p. 76-77) dahin äusserte, dass der Ausdruck der $\boldsymbol{P}$-Function durch ein bestimmtes Integral zur Bestimmung der in den Fundamentalzweigen $P^{\lambda^{\prime}}, P^{\lambda^{\prime \prime}}, \ldots$ noch willkürlich gebliebenen Factoren benutzt werden solle. Allerdings benutzte Riemann ja keine homogenen Variabelen; ich nehme an, dass er eben hierdurch gehindert war, die Sache zu einem gaten Abschluss zu bringen. 
die $x_{1}, x_{2}$ sind also in dem $\Pi_{1}, \Pi_{2}$ vom Grade:

$$
2:\left(\frac{1}{l}+\frac{1}{m}+\frac{1}{n}-1\right) \text {. }
$$

Es ist dies die zuerst von Halphen gefundene Zahl (Comptes Rendus, t. $\left.92(1881, \mathrm{I}))^{*}\right)$. Die neue Ableitung, welche hier für diese Zahl gegeben ist, oder vielmehr die neue Bedeutung, unter der dieselbe hier auftritt, dürfte unter principiellen Gesichtspunkten bemerkenswerth sein.

Göttingen, den 23. December 1890.

*) Vergl. die bez. Erläuterungen auf p. 129 des Bandes I meiner von Herrn Fricke herausgegebenen Vorlesungen über elliptische Modulfunctionen (Leipzig 1890). 